\title{
Alergia a fórmula de aminoácidos libres en síndrome de intestino corto - Reporte de 2 casos
}

\section{Two Case Reports of Allergies to Amino Acid Based Formula in Patients with Short Bowel Syndrome}

Mónica Contreras Ramírez, MD, ${ }^{1}$ Catalina Ortiz-Piedrahita, MD, ${ }^{1}$ Lina María Castaño Jaramillo, MD, ${ }^{2}$ Ana Elvira Montero Carvajalino, MD, ${ }^{3}$ Juan Camilo Pérez Cadavid, MD ${ }^{4}$

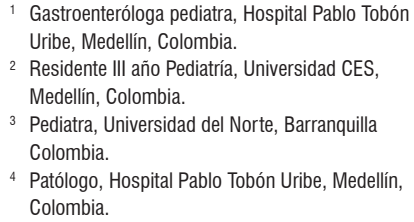

3 Pediatra, Universidad del Norte, Barranquilla Colombia.

4 Patólogo, Hospital Pablo Tobón Uribe, Medellín, Colombia.

\begin{abstract}
Resumen
El síndrome de intestino corto constituye una entidad con alteración en la superficie de absorción y en la funcionalidad del sistema gastrointestinal. En estos pacientes se ha descrito mayor predisposición de alergias alimentarias a la proteína de la leche de vaca y al huevo. Se describen los casos de 2 lactantes menores con síndrome de intestino corto y reacciones alérgicas a fórmulas de aminoácidos libres. En el primer caso la paciente presentó una respuesta no mediada por inmunoglobulina $E(\lg E)$ manifestada clínicamente con una colitis eosinofílica, que mejoró con uso de esteroides sistémicos, cambio a otra fórmula elemental. En el segundo caso se presenta una paciente con intolerancia inicial a la leche materna y fórmula extensamente hidrolizada. Con primera fórmula de aminoácidos libres presenta sangrado gastrointestinal y manifestaciones cutáneas, por lo que se hace cambio a otra fórmula de aminoácidos libres con tolerancia transitoria. Subsecuentemente, en el contexto de un cuadro viral no especificado con compromiso respiratorio y gastrointestinal, presenta reacción anafiláctica a la fórmula de aminoácidos libres que venía tolerando previamente, por lo que se hace necesario cambio a dieta modular con tolerancia adecuada. No se encontraron reportes previos en la literatura de reacciones alérgicas a fórmulas elementales a base de aminoácidos, por lo cual se reportan los casos descritos. Aún no hay consenso en cuanto a la fórmula ideal para iniciar la alimentación en los pacientes con intestino corto para evitar sensibilización alergénica.
\end{abstract}

\section{Palabras clave}

Alergia alimentaria, síndrome de intestino corto, fórmula elemental, aminoácidos.

\section{Abstract}

Short bowel syndrome (SBS) is characterized by alterations in the absorption surface and in the functionality of the gastrointestinal system. Patients with SBS are more prone to allergies the protein in cow's milk and eggs. We report two cases of infants with SBS and allergic reactions to amino acid based formulas. In the first case, the patient's response was not IgE-mediated. It manifested clinically with eosinophilic colitis that improved with the use of systemic steroids and a change to another elemental formula. In the second case, the patient initially presented intolerance to breast milk and extensively hydrolyzed formula. After feeding with the first free amino acid formula, the patient developed gastrointestinal bleeding and skin manifestations. Another free amino acid formula was substituted. The patient had transient tolerance to the new formula. Later, in the context of an unspecified viral infection with respiratory and gastrointestinal involvement, the patient presented an anaphylactic reaction to the formula which had previously been tolerated. This necessitated a change to a modular diet which was adequately tolerated. We have presented this case report because no previous reports were found in the literature about allergic reactions to basic amino acid-based formulas. There is still no consensus on the ideal formula for preventing allergic sensitization in patients with SBS.

\section{Keywords}

Food allergy, short bowel syndrome, elemental formula, amino acids. 


\section{INTRODUCCIÓN}

El síndrome de intestino corto (SIC) es una patología secundaria a la resección intestinal, que conlleva a menor superficie de absorción con alteraciones metabólicas y nutricionales. Después de la resección, el intestino remanente inicia un proceso de adaptación con múltiples cambios paulatinos estructurales y funcionales. Esta condición puede predisponer a la sensibilización por diferentes alérgenos, vía intestinal, y riesgo aumentado de alergias alimentarias, como se ha reportado en algunos estudios $(1,2)$. Se describe el caso de dos lactantes con SIC, que presentaron reacciones alérgicas durante la administración de alimentación enteral con fórmulas de aminoácidos libres (FA), las características principales se presentan en la Tabla $\mathbf{1}$.

\section{PRESENTACIÓN DE CASOS CLÍNICOS}

\section{Caso 1}

Paciente femenina de 6 meses, con antecedente de prematuridad y atresia intestinal. Desde el nacimiento fue alimentada con FA (Puramino Mead-Johnson y después con Neocate Nutricia). A los 5 meses de vida presenta curva estacionaria de peso y deposiciones con moco y sangre, sin evidencia de etiología infecciosa (rotavirus, adenovirus, parásitos en materia fecal y coprocultivo negativos). La endoscopia digestiva y colonoscopia evidenció aftas en duodeno, colon sigmoides y recto. Las biopsias revelaron cambios inflamatorios con eosinófilos de $35 \times$ CAP en recto (Figura 1), sin hallazgos de eosinofilia periférica. Se manejó con reposo intestinal y esteroides sistémicos, con resolución de los síntomas. Posteriormente se reinicia alimentación con FA (Puramino Mead-Johnson) sin recurrencia del cuadro.

\section{Caso 2}

Paciente femenina de 23 meses con antecedente de prematuridad y atresia intestinal. Al mes de vida se inicia alimentación enteral con leche materna y presenta vómito y hematoquecia (rectorragia), por lo tanto se inicia fórmula extensamente hidrolizada (FEH) (Nutramigen Mead Johnson) y dieta de exclusión materna. Posteriormente requirió cambio a FA (Neocate ${ }^{\circ}$ Nutricia) por persistir sintomática y evidencia de eosinófilos en heces del $25 \%$. Luego de 2 meses de tolerancia a la misma, presentó síntomas gastrointestinales, hematoquecia y exantema ( $r a s h$ ) urticariforme, por lo que se cambió a otra FA (Puramino Mead-Johnson), la cual toleró hasta los 23 meses. Consulta nuevamente por cuadro de origen viral con compromiso gastrointestinal y respiratorio, durante el cual presenta reacción anafiláctica (urticaria, taquicardia, dificultad respiratoria y sibilancias) tras la administración de Puramino (Figura 2), que se resolvieron con dosis única de adrenalina intramuscular. Se indicó dieta de exclusión de FA con adición de módulos de maltodextrinas y aceite (Farmal + aceite de oliva) sin recurrencia del cuadro alérgico.

\section{DISCUSIÓN}

En el SIC, además de afectarse la absorción, se alteran otras funciones como la pérdida de tejido linfoide asociado a mucosas, lo que facilita el sobrecrecimiento bacteriano, la

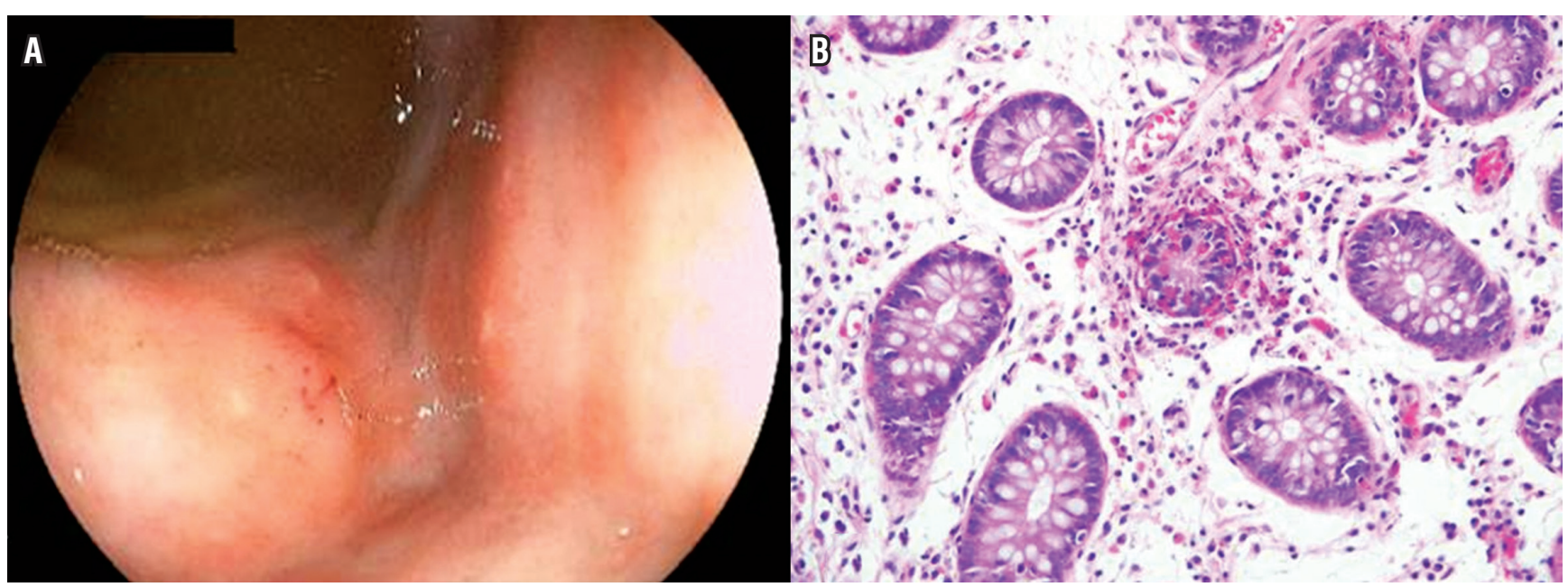

Figura 1. A. Colonoscopia de paciente 1 con aftas en unión rectosigmoidea. B. Coloración hematoxilina eosina $400 \mathrm{x}$ de mucosa rectal con lámina propia edematosa, infiltrado inflamatorio linfoplasmocitario y presencia de numerosos eosinófilos que forman acúmulos y penetran al epitelio de las criptas. 
inflamación intestinal y el incremento de la permeabilidad de la mucosa a los antígenos de la dieta, con el riesgo consecuente de sensibilización a los mismos. Otros factores como la prematuridad también se han asociado a mayor riesgo de alergia alimentaria (1-3). Se presentan 2 casos de lactantes con antecedente de prematuridad e intestino corto, secundario a resección quirúrgica por atresia intestinal que desarrollaron síntomas clínicos (digestivos y cutáneos) de alergia alimentaria, corroborada con evidencia de infiltración eosinofílica tisular en uno de los casos y mejoría con exclusión de la fórmula de aminoácidos en ambos casos.

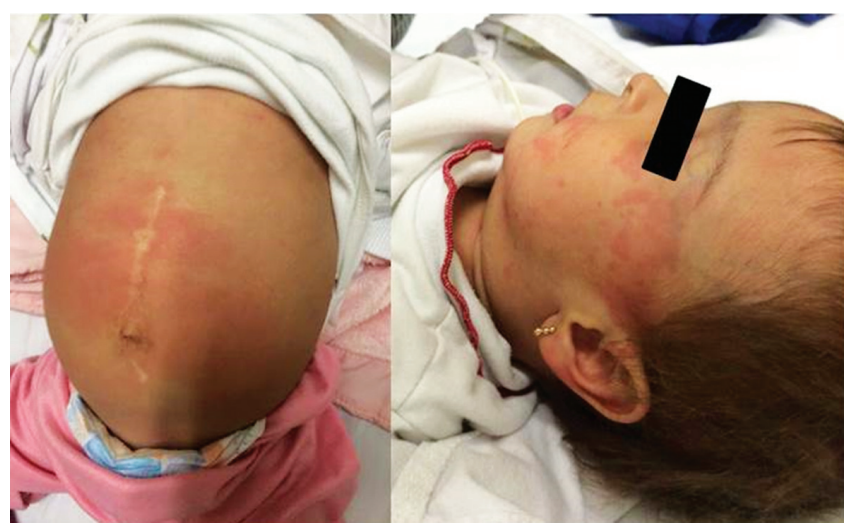

Figura 2. Paciente caso 2 con urticaria secundaria a administración de Puramino.

En nuestra institución no contamos con la prueba de hidrógeno espirado para el diagnóstico de sobrecrecimiento bacteriano, por lo que se realizan ciclos de antibióticos intraluminales (metronidazol y/o rifaximina), en caso de sospecha clínica, lo cual se hizo en ambas pacientes, pero no concomitante con la aparición de los síntomas clínicos.

No hay consenso con respecto a la fórmula ideal para iniciar la alimentación enteral en los pacientes con intestino corto. Estudios en modelos animales con SIC han reportado mejor tolerancia con el uso de fórmulas de aminoácidos libres (4). Estudios en humanos no evidencian diferencias en la absorción de proteínas, permeabilidad y adaptación intestinal al utilizar FEH o una fórmula de proteína entera $(5,6)$. Sin embargo, algunos autores han propuesto el uso de hidrolizados de leche en los pacientes menores de dos años con SIC debido a la mayor susceptibilidad de estos a la inflamación de la mucosa que pudiera desencadenar sensibilización y enfermedad alérgica gastrointestinal (7). En nuestro hospital para los niños con intestino corto se inicia FEH y sólo en caso de intolerancia o síntomas de alergia se hace cambio a FA.

Cabe resaltar que nuestros 2 casos estaban recibiendo FA de forma prolongada en el momento de la aparición de los síntomas. Una de las pacientes presentó el episodio alérgico después de una infección viral aguda. Ninguno de los artículos previamente mencionados reportan la aparición de alergia alimentaria con el uso de fórmulas elementales. En nuestras pacientes no hay historia de consumo previo de proteína de leche de vaca (PLV) entera que pudiera dar origen a la sensibilización; excepto en el segundo caso, donde la paciente tuvo historia de ingesta de leche materna por corto tiempo durante el período neonatal.

Algunos autores sugieren que el uso prolongado de FA puede llevar a atrofia de la mucosa intestinal, lo cual, en condiciones de estrés, incrementa su permeabilidad y aumenta el riesgo de sensibilización a macromoléculas de origen

Tabla 1. Características de las pacientes

\begin{tabular}{lll}
\hline \multicolumn{1}{c}{ Características } & Caso 1 & Caso 2 \\
\hline Causa del SIC & Atresia intestinal & Atresia intestinal \\
Intestino residual & 10 centímetros & 60 centímetros \\
Presencia de válvula ileocecal & No & No \\
Edad de inicio síntomas de alergia & 5 meses & 23 meses \\
Duración de fórmula elemental antes de inicio & 5 meses & 20 meses \\
de síntomas & & \\
Estudios diagnósticos & Endoscopia y colonoscopia con aftas en & Prick to prick para látex y Neocate: Negativo \\
& duodeno, colon sigmoides y recto. Biopsias: & RAST para trofoalergenos: negativo. \\
Mecanismo de alergia & Eosinófilos hasta 35 x CAP en recto. & \\
\hline
\end{tabular}

Datos clínicos de paciente con SIC con alergia a fórmula elemental.

CAP: campo de alto poder; RAST: Radi oallergo sorbent test; SIC: síndrome intestino corto. 
alimentario (5). En el caso 1 no se documentó atrofia intestinal en la endoscopia, sin embargo el compromiso atrófico puede ser parcheado, lo que no excluye este diagnóstico en la paciente. En el caso 2 no se realizaron estudios endoscópicos para buscar atrofia intestinal o infiltración tisular debido a la mejoría significativa con la dieta de exclusión de FA. Otra posibilidad que explicaría las reacciones alérgicas es la exposición a trazas de proteínas diferentes a la PLV, que pueden estar presentes en el aceite utilizado en la composición de estas fórmulas (2).

La alergia al huevo y a la proteína de leche de vaca (PLV) en los niños con SIC ha sido descrita en la literatura. Leechawengwongs y colaboradores reportan el caso de dos pacientes con SIC, evidencia clínica y paraclínica de alergia a PLV y otros alérgenos (3). Masumoto describe dos lactantes con SIC ultracorto y síntomas alérgicos al iniciar fórmula con proteína entera de la leche de vaca (2). Mazon y colaboradores reportan una serie de 14 niños con SIC, de los cuales $57 \%$ presentaron sensibilización a la leche de vaca, con pruebas de alergia, prueba de punción (prick test) e inmunoglobulina E (IgE) específica por inmunocap positivas; estos pacientes solo habían recibido alimentación con FEH y no tenían antecedente familiar de enfermedad alérgica (1). Diamanti describe el caso de 3 pacientes con SIC, todos con evidencia de APLV tras la introducción de PLV con pruebas de alergia positivas (8). En el caso 2 las pruebas de alergia IgE mediadas fueron negativas para trofoalergénos y una de las fórmulas de aminoácidos lo que hace difícil el diagnóstico de la alergia; como lo describió Mazon y colaboradores, donde el $43 \%$ de los pacientes con alergia alimentaria tenían pruebas IgE mediadas negativas. La presentación de estos casos clínicos deja abierta la discusión con respecto a qué tipo de fórmulas usar en pacientes con SIC para evitar la aparición de sensibilización a PLV, principalmente en condiciones de estrés como las descritas anteriormente.

\section{REFERENCIAS}

1. Mazon A, Solera E, Alentado N, Oliver F, Pamies R, Caballero $\mathrm{L}$, et al. Frequent IgE sensitization to latex, cow's milk, and egg in children with short bowel syndrome. Pediatr Allergy Immunol Off Publ Eur Soc Pediatr Allergy Immunol. 2008 Mar;19(2):180-3.

2. Masumoto K, Esumi G, Teshiba R, Nagata K, Hayashida M, Nakatsuji T, et al. Cow's milk allergy in extremely short bowel syndrome: Report of two infants. Eur E-J Clin Nutr Metab. 2008;3(5):e217-9.

3. Leechawengwongs E, Tison BE, Gopalakrishna GS, Reid BS, Bacino CA, Haws AL, et al. Does short bowel syndrome increase the risk of food allergy and eosinophilic gastrointestinal disease? Observations in Shah-Waardenburg syndrome. J Allergy Clin Immunol. 2013;131(1):251-5.

4. Hua Z, Turner JM, Mager DR, Sigalet DL, Wizzard PR, Nation PN, et al. Effects of polymeric formula vs elemental formula in neonatal piglets with short bowel syndrome. JPEN J Parenter Enteral Nutr. 2014;38(4):498-506.

5. Ksiazyk J, Piena M, Kierkus J, Lyszkowska M. Hydrolyzed versus nonhydrolyzed protein diet in short bowel syndrome in children.J Pediatr Gastroenterol Nutr. 2002;35(5):615-8.

6. Olieman JF, Penning C, Ijsselstijn H, Escher JC, Joosten KF, Hulst JM, et al. Enteral nutrition in children with shortbowel syndrome: current evidence and recommendations for the clinician. J Am Diet Assoc. 2010;110(3):420-6.

7. Vanderhoof JA, Young RJ. Hydrolyzed versus nonhydrolyzed protein diet in short bowel syndrome in children. J Pediatr Gastroenterol Nutr. 2004;38(1):107.

8. Diamanti A, Fiocchi AG, Capriati T, Panetta F, Pucci N, Bellucci F, et al. Cow's milk allergy and neonatal short bowel syndrome: comorbidity or true association? Eur J Clin Nutr. 2015;69(1):102-6. 